\title{
Posttransplant de novo donor specific HLA antibody monitoring and clinical outcomes: a single-center experience
}

\author{
Nurettin $A^{1}{ }^{1}, \operatorname{Vahhac}^{\mathrm{Alp}}{ }^{1} \oplus$, Şeymus Kaya ${ }^{2} \odot$ \\ ${ }^{1}$ Department of General Surgery, Organ Transplant Center, University of Health Sciences, Gazi Yaşargil Training and Research \\ Hospital, Diyarbakir, Turkey \\ ${ }^{2}$ Department of Pathology, University of Health Sciences, Gazi Yaşargil Training and Research Hospital, Diyarbakır, Turkey
}

\begin{abstract}
Objectives: Despite the improvements in early-term outcomes of kidney transplantation, late-term graft failure still remained as a critical problem. De novo donor specific antibodies (DSA) developing against direct human leukocyte antigens (HLA) are the significant risk factors for shortened graft survival in the previously nonsensitized cases. The purpose of this study is to evaluate the clinical outcomes of de novo DSA development in the kidney transplant cases.

Methods: The present study included 121 (alive/cadaver: 106/15) of 148 (alive/cadaver: 125/23) cases who were not previously sensitized (PRA and DSA negative) and undergone kidney transplantation between August 2012-January 2018. DSAs of the cases without expected declines in creatinine levels in the polyclinic followups and postoperative early-term were evaluated. Renal biopsy was performed in the cases encountered with $>2000$ mean fluorescence intensity (MFI) de novo DSA against HLA-A, HLA-B, HLA-DR. Treatment protocol of plasmapheresis+intravenous immunoglobulin (IVIG)+rituximab (in the cases without clinical response) was administered in the cases with antibody-mediated rejection (AMR) detected by renal biopsy. In addition, the presence of de novo non-DSA was also evaluated in the cases. The presence of de novo was encountered by identifying the specificities of anti-HLA antibody specificities using Luminex single antigen beads in the recipient serum.
\end{abstract}

Results: De novo DSA (antibodies against HLA-A, HLA-B, HLA-DR and HLA-DQ) were monitored in 23 cases. DQ positivity was detected in 10 cases. MFI values were $>4000$ and 2000-4000 in 8 and 2 cases, respectively. De novo non-DSA was found in 19 cases. Biopsy was performed in 8 cases due to the development of MFI > 2000 de novo DSA against HLA-A, HLA-B and HLA-DR and the findings of acute humoral rejection (AHR) were encountered in 2 cases. Additionally, acute humoral rejection was diagnosed in 1 case that developed de novo non-DSA. Two cases were diagnosed with AHR by biopsy although no de novo DSA or non-DSA developed and renal graft loss occurred in these two cases.

Conclusions: The fact that routine DSA monitoring in all the cases provided no significant contribution to the outcomes of our study may contribute to the debates on the necessity of DSA monitoring in the patients with low immunological risk.

Keywords: De novo DSA, kidney, transplantation, monitoring

How to cite this article: Ay N, Alp V, Kaya S. Posttransplant de novo donor specific HLA antibody monitoring and clinical outcomes: a single-center experience. Eur Res J 2021;7(3):304-311. DOI: 10.18621/eurj.732625

Address for correspondence: Nurettin Ay, MD., University of Health Sciences, Gazi Yaşargil Training and Research Hospital, Department of General Surgery, Organ Transplant Center, Diyarbakır,Turkey.E-mail: nurettinay77@hotmail.com,Tel: +90412 2580074/2348 
D espite the improvements in early-term outcomes of kidney transplantation, late-term graft failure still remained as a critical problem [1]. Current studies demonstrated that antibody-mediated humoral tissue injury is responsible for more $60 \%$ of the late graft losses [2-4]. De novo donor specific antibodies (DSA) developing against direct human leukocyte antigens (HLA) are the significant risk factors for shortened graft survival in the previously non-sensitized cases. The presence of DSA may be associated with antibody-mediated rejection (AMR) and cause shortened graft survival [5-7]. That fact orientated the researchers to investigate the HLA antibodies $[8,9]$. The purpose of the present study is to evaluate the clinical outcomes of de novo DSA development in the cases who underwent kidney transplantation.

\section{METHODS}

The present study included 121 (living/deceased: 106/15) of 148 (living/deceased: 125/23) caseswhowere not previouslysensitized (PRA and DSA negative) and undergone kidney transplantation between August 2012-January 2018. Ethics committee approval dated 17.01.2017 No:91 of the study was obtained from the Ethics Committee of University of Health Sciences Gazi Yaşargil Training and Research Hospital. All transplantations were performed after achieving negative T-cell crossmatch by flow cytometry and complement dependent cytotoxicity (CDC) methods. The cases without expected decline in the creatinine levels in the postoperative early term and those who received routine DSA assessment once at every 3 months for the first year and then once yearly were included in the study. The cases in whom this protocol could not be implemented were excluded from the study. Renal biopsy was performed in the cases encountered with $>2000$ mean fluorescence intensity (MFI) de novo DSA against HLA-A, HLA-B and HLA-DR. Renal biopsies were evaluated according to the present Banff criteria at the time of biopsy. Treatment protocol of plasmapheresis+intravenous immunoglobulin (IVIG)+rituximab (in the cases without clinical response) was administered in the cases with humoral rejection detected by renal biopsy. In addition, the presence of de novo non-DSA was also evaluated in the cases. The presence of de novo
DSAwas encountered by identifying the specificities of anti-HLA antibody specificities using Luminex single antigen beads in the recipient serum. The demographic and clinical characteristics of the patients were presented in Table 1.

\section{Immunosuppression and Prophylaxis}

Basiliximab (20 mg/day; at the day of operation and postoperative 4th day) and antithymocyteglobulin (ATG; for high-risk patients; $3 \mathrm{mg} / \mathrm{kg}$ during operation and $1.5 \mathrm{mg} / \mathrm{kg}$ at the postoperative $1 \mathrm{st}$ and $2 \mathrm{nd}$ days) were administered as the induction therapy. Methylprednisolone $1000 \mathrm{mg}$ was administered intraoperatively. This dose was gradually tapered in the following days and switched to $20 \mathrm{mg}$ oral prednisolone at the postoperative 6th day. Oral prednisolone dosage was reduced gradually to reach $5 \mathrm{mg}$ a day at the first year after transplantation. Calcineurin inhibitors (CNI; tacrolimus: 0.1-0.15 mg/kg/day and cyclosporine: $6-8 \mathrm{mg} / \mathrm{kg} /$ day) and mycophenolate mofetil (MMF; 2g/day, by splitting into two doses) or Mycophenolate sodium (MMF; $1440 \mathrm{mg} /$ day, by splitting into two doses) were administered for maintenance of the immunosuppression. MMF dose of 600 $\mathrm{mg} / \mathrm{m}^{2}$ was administered as split into two doses in the children. Everolimus (replaced by MMF at the 5th day) was added to the treatment protocol to be used together with tacrolimus in the cases with $2 \geq$ mismatches. Target level of everolimus with tacrolimus was attempted to maintain between $4-8 \mathrm{mg} / \mathrm{dL}$. However, everolimus was used instead of CNI in the cases supposed to develop thrombotic microangiopathy (TMA) because of use of CNIs and target level was attempted to maintain between 8-10 mg/dL. Trimetho-

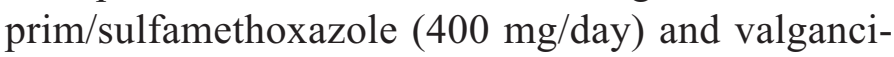
clovir (900 mg/day) were administered for the prophylaxis of Pneumocystis Jirovecii (until the postoperative 6th month) and cytomegalovirus (CMV) (until the postoperative 3rd month), respectively. Delayed graft function (DGF) was defined as the need for dialysis in the posttransplant period. Graft loss was defined as return of the recipient to dialysis due to graft failure. Acute rejection was diagnosed by renal biopsy. Acute cellular rejection (ACR) was treated with intravenous pulse methylprednisolone and/or ATG therapy depending on the severity of rejection. The cases who were diagnosed or comorbid with AMR received the treatment of plasmapheresis+intra- 
Table 1. Descriptive data of the kidney transplant cases

\begin{tabular}{|c|c|}
\hline Characteristics & Data \\
\hline \multicolumn{2}{|l|}{ Gender $\mathrm{M} / \mathrm{F}$} \\
\hline Recipient & $69 / 52(57 \% / 43 \%)$ \\
\hline Donor & $41 / 65(39 \% / 61 \%)$ \\
\hline \multicolumn{2}{|l|}{ Age (years) } \\
\hline Recipient & $34.1(11-68)$ \\
\hline Donor & 41 (10ay-71) \\
\hline Follow-up period (months) & $32.5(1-68)$ \\
\hline Living donor/deceased donor & $106 / 15(87.6 \% / 12.4 \%)$ \\
\hline \multicolumn{2}{|l|}{ Operation of donor $(\mathrm{O} / \mathrm{L})$} \\
\hline Open nephrectomy & $81(67 \%)$ \\
\hline Laparoscopic nephrectomy & $40(33 \%)$ \\
\hline \multicolumn{2}{|l|}{ Relationship } \\
\hline Spouse & $35(33 \%)$ \\
\hline First degree & $41(39 \%)$ \\
\hline Second degree & $19(18 \%)$ \\
\hline Third degree & $3(3 \%)$ \\
\hline Fourth degree & $2(2 \%)$ \\
\hline Unrelated & $6(5 \%)$ \\
\hline Number of DSA studied patients & $121(81.7 \%)$ \\
\hline Median DSA positive time & $21(4-56)$ \\
\hline Preemptive & $38(36 \%)$ \\
\hline \multicolumn{2}{|l|}{ Induction } \\
\hline None & $15(13 \%)$ \\
\hline ATG & $67(55 \%)$ \\
\hline Basiliximab & $39(32 \%)$ \\
\hline Incompatibility numbers & $15(12.3 \%)$ \\
\hline 1 & $4(3.3 \%)$ \\
\hline 2 & $17(14 \%)$ \\
\hline 3 & $42(34.7 \%)$ \\
\hline 4 & $15(12.4 \%)$ \\
\hline 5 & $17(14 \%)$ \\
\hline 6 & $11(9 \%)$ \\
\hline \multicolumn{2}{|l|}{ Rejection } \\
\hline Acute celluler & $4(2.7 \%)$ \\
\hline Acute humoral & $4(2.7 \%)$ \\
\hline Celluler + Humoral & $1(0.6 \%)$ \\
\hline Graft loss & $6(4 \%)$ \\
\hline CAN & 1 \\
\hline Humoral rejection & 2 \\
\hline $\mathrm{BKN}$ & 1 \\
\hline RAP & 1 \\
\hline FSGS recurrence & 1 \\
\hline Death & $2(1.6 \%)$ \\
\hline DGF & $3(2.4 \%)$ \\
\hline Discharge creatinine (mg/dl) & $1.14(0.55-2.4)$ \\
\hline Last creatinine level (mg/dl) & $1.1(0.49-4.34)$ \\
\hline Length of hospital stay (days) & $10.1(5-42)$ \\
\hline
\end{tabular}


venous immunoglobulin (IVIG) every other day.

\section{Statistical Analysis}

Statistical analysis was performed using SPSS Software Version 16. The probability of the variables for normal distribution was tested by analytic statistical methods (Kolmogorov-Smirnov/Shapiro-Wilk tests). Descriptive analyses were given as median values for non-normally distributed variables. Since study data were non-normally distributed between the groups (DSA, Non-DSA and non-anti-HLA); creatinine values were compared using Mann-Whitney $\mathrm{U}$ test. A p-value less than 0.05 was accepted as statistically significant.

\section{RESULTS}

Mean follow-up duration ranged between 1 and 68 months in 121 cases included in the study. Median follow-up period was 32.5 months. HLA antibodies were encountered in 42 (34.7\%; de novo DSA: 23 , de novo Non-DSA: 19) cases. De novo DSA (DSA-A, DSA-B and DSA-DR) were detected in 10 cases. De novo DSA MFI value was $>2000$ in $8(6.6 \%)$ of those cases. We have determined DQ positivity in 13 $(10.7 \%)$ cases. MFI values were $>4000,2000-4000$ and 1000-2000 in 8, 2 and 3 cases with DQ positivity, respectively (Table 2 ). In the postoperative term, de novo DSA and de novo non-DSA developed in 21 th (a range of 4-56 months) and 24th (a range of 4-45 months) months, respectively. However, no statistically significant difference was present between the times of development of de novo DSA also in the postoperative term. In addition, no statistically significant correlation was detected between development of DSA and recipient age, recipient gender, DGF and number of donor-recipient matches. Except the cases with occurrence of graft loss, recent creatinine levels of the patients without anti-HLA antibodies (non antiHLA), with de novo DSA and de novo non-DSA patients were $1.18 \pm 0.51(0.50-4.34), 1.30 \pm 0.58$ (0.89-2.75), and $1.01 \pm 0.28(0.49-1.6)$, respectively. No statistically significant difference was found between the creatinine levels of the patients with non anti-HLA compared with de novo DSA and de novo non-DSA ( $p=0.819$ and $p=0.401$, respectively). No statistically significant difference was detected also
Table 2. De novo DSA and de novo Non-DSA data

\begin{tabular}{|cc}
\hline & $\mathbf{n ~ ( \% )}$ \\
\hline De novo DSA (A, B, DR) & $111(91.7 \%)$ \\
\hline DSA NEGATIVE & $2(1.6 \%)$ \\
$1000<$ CLASS $2<2000$ & $5(4.1 \%)$ \\
\hline CLASS $1>2000$ & $2(1.6 \%)$ \\
CLASS $2>2000$ & $1(0.8 \%)$ \\
\hline CLASS 1 and 2 $>2000$ & \\
De novo DSA (DQ) & $3(2.5 \%)$ \\
De novo Non-DSA & $2(1.6 \%)$ \\
DQ CLASS 2 $>4000$ & $8(6.6 \%)$ \\
2000 $<$ DQ CLASS $2<4000$ & $19(15.7 \%)$ \\
\hline $1000<$ DQ CLASS $2<2000$ &
\end{tabular}

DSA $=$ Donor specific antibody

between the creatinine levels of the patients with de novo DSA and de novo non-DSA $(p=0.189)$. The findings of AMRwas encountered in 2 of the 8 patients with de novo DSA by renal biopsy (Table 3) (Cases 3 and 6). These two cases became DSA negative after plasmapheresis+intravenous immunoglobulin (IVIG) therapy. However they were found DSA positive again during their follow-ups. In the follow-ups; 3 of 6 DSA positive cases became negative without implementation of any treatment. The rest 3 cases remained positive. Besides, one case with develoment of non-DSA was diagnosed with AMR. AMR developed in one patient without de novo DSA and one patient with de novo DSA DQ positivity (Table 3) (Cases A, B and C).

\section{DISCUSSION}

Although many studies have determined the association between development of de novo DSA after kidney transplantation and an immunological phenomenon such as AMR, there is no consensus on routine DSA monitoring in the posttransplantation period. Routine DSA monitoring is not performed in the postoperative follow-ups in many centers. In our study, we have evaluated posttransplant DSA outcomes of 121 non-sensitized cases (the cases that do not require implementation of desensitization protocol in the preoperative period) and the association between these outcomes and AMR. HLA-antibodies were encountered in $42(34.7 \%)$ cases. De novo DSA was detected in 23 (17.4\%; antibodies against HLA-A, HLA-B, 
Table 3. De novo DSA/Non-DSA and acute humoral rejection relationship with cases

\begin{tabular}{|c|c|c|c|c|c|c|}
\hline Cases & $\begin{array}{l}\text { DSA type } \\
\text { and } \\
\text { MFI values }\end{array}$ & $\begin{array}{c}\text { MM } \\
\text { count }\end{array}$ & Induction & $\begin{array}{l}\text { Biopsy } \\
\text { result }\end{array}$ & $\begin{array}{c}\text { De novo } \\
\text { DSA/non- } \\
\text { DSA process }\end{array}$ & Treatment \\
\hline 1 & $\begin{array}{c}\text { Class I } \\
3213 \\
\text { Class II } \\
7428\end{array}$ & 4 & ATG & $\begin{array}{l}\text { The patient } \\
\text { did not give } \\
\text { approval }\end{array}$ & 56th month & $\begin{array}{l}\text { Cr level is stable } \\
\text { Outpatient clinic } \\
\text { follow-up }\end{array}$ \\
\hline 2 & $\begin{array}{l}\text { Class I } \\
11089\end{array}$ & 4 & ATG & CAN & 51th month & $\begin{array}{l}\text { Outpatient clinic } \\
\text { follow-up }\end{array}$ \\
\hline 3 & $\begin{array}{l}\text { Class II } \\
20182\end{array}$ & 3 & Basiliximab & AHR & 24th month & $\begin{array}{c}\text { PE + IVIG } \\
\text { Outpatient clinic } \\
\text { follow-up }\end{array}$ \\
\hline 4 & $\begin{array}{c}\text { Class II } \\
3441\end{array}$ & 3 & Basiliximab & CAN & 12 th month & $\begin{array}{l}\text { Outpatient clinic } \\
\text { follow-up }\end{array}$ \\
\hline 5 & $\begin{array}{c}\text { Class I } \\
4336\end{array}$ & 4 & ATG & No rejection & 26th month & $\begin{array}{l}\text { Outpatient clinic } \\
\text { follow-up }\end{array}$ \\
\hline 6 & $\begin{array}{l}\text { Class I } \\
6583\end{array}$ & 4 & ATG & AHR & 18th month & $\begin{array}{l}\text { Outpatient clinic } \\
\text { follow }\end{array}$ \\
\hline 7 & $\begin{array}{l}\text { Class I } \\
2356\end{array}$ & 0 & No induction & CAN & 8th month & $\begin{array}{l}\text { Outpatient clinic } \\
\text { follow }\end{array}$ \\
\hline 8 & $\begin{array}{l}\text { Class I } \\
9482\end{array}$ & 0 & ATG & No rejection & 4th month & $\begin{array}{l}\text { Outpatient clinic } \\
\text { follow }\end{array}$ \\
\hline $\mathbf{A}$ & $\begin{array}{c}\text { De novo DSA } \\
(-)\end{array}$ & 2 & Basiliximab & AHR & $\begin{array}{l}\text { De novo } \\
\text { DSA (-) }\end{array}$ & $\begin{array}{c}\text { PE + IVIG; } \\
\text { Graft loss }\end{array}$ \\
\hline B & $\begin{array}{l}\text { Class II } \\
9654\end{array}$ & 3 & ATG & AHR & $\begin{array}{c}\text { De novo } \\
\text { DSA (+ } \\
\text { DQ) }\end{array}$ & $\begin{array}{c}\text { PE + IVIG } \\
\text { Outpatient clinic } \\
\text { follow-up }\end{array}$ \\
\hline $\mathbf{C}$ & $\begin{array}{c}\text { Class I } \\
32227 \\
\text { Class II } \\
37124\end{array}$ & 3 & ATG & $\begin{array}{l}\operatorname{ACR}(+) \\
\operatorname{AHR}(+)\end{array}$ & $\begin{array}{c}\text { De novo } \\
\text { DSA (-) } \\
\text { De novo non- } \\
\text { DSA }(+)\end{array}$ & $\begin{array}{c}\text { PE + IVIG } \\
\text { Graft loss }\end{array}$ \\
\hline
\end{tabular}

$\mathrm{AHR}=$ Acute Humoral Rejection, DSA $=$ Donor specific antibody, ATG $=$ Anti-tymocyte globülin $\mathrm{Cr}=$ Creatinine, $\mathrm{CAN}=$ Chronic allograft nephropathy, $\mathrm{ACR}=$ Acute cellular rejection, $\mathrm{PE}=$ Plasma exchange, IVIG = Intravenous immunoglobulin, MFI = Mean Fluorescence Intensity

HLA-DR and HLA-DQ) cases. DQ positivity was determined in $13(10.7 \%)$ of those cases. The findings of AMR was observed in 2 of 8 cases with de novo DSA development. In addition, a case with de novo non-DSA development was diagnosed with AMR. AMR was also diagnosed by biopsy in two cases despite absence of de novo DSA development and graft loss occurred in these cases. A significant association of de novo DSA development with graft loss and re- duced graft survival has been found also in the literature. Li et al. [10] have determined that 1-, 3- and 5year survival rates of the patients with de novo DSA development were $92 \%, 77 \%$ and $69 \%$, respectively. Whereas, these rates were $100 \%, 100 \%$ and $96 \%$ in the cases without de novo DSA development, respectively. The same study has encountered that occurrence of de novo DSA was not correlated with development of AMR with in the posttransplant first 
6 months whereas occurrence of de novo DSA was found significantly correlated with development of AMR after posttransplant 6th month [10]. Time interval between the occurrence of posttransplant de novo DSA and development of rejection still remained unclear. The previous studies have suggested that de novo DSA development has no negative effect on graft function [11, 12]. Terasaki et al. [12] have carried out a 1-year prospective follow-up study in 23 centers and found that the rates of graft failure in the cases with and without development of anti-HLA antibodies were $6.6 \%$ and $3.3 \%$, respectively. In the same manner, Süsal et al. [13] have emphasized the importance of postoperative DSA monitoring in the patients with high immunological risk and impaired graft function. However, debates on DSA are still ongoing. Although, some studies have stated that DSA positivity in the absence of rejection has no impact on graft survival, there are also other studies which have noted that early and late developments of DSA have similar impact on graft injury [14, 15]. Prajuli et al. [14] has denoted in his study that DSA positivity in the absence of rejection has no impact on graft survival. In that study, post-biopsy follow-up period was shorter than 3 years. That period may be inadequate to draw reasonable conclusions. In our study, 2 cases with DSA development were diagnosed with biopsy-proven AMR. However, 2 cases without detection of DSA were also diagnosed with AMR by biopsy. Non DSA was present in one of those cases and graft loss occurred in both cases. Another case in our study was HLA-DQ DSA-positive and diagnosed with AMR by renal biopsy. Even though, there is no consensus on DSA positivity and graft survival with all aspects in the literature, our outcomes are not exactly consistent with general literature. That may be resulting from the fact that number of DSA positive patients is not high enough to present significant outcomes. Ginevri et al. [16] have identified de novo DSA and de novo nonDSA in respectively $19(23 \%)$ and $24(29 \%)$ patients in their 4.3-year follow-up study on 82 pediatric cases with kidney transplantation. Median DSA development duration was 24 months and DSAs were mostly against HLA-DQ antigens. Of the 82 cases; 8 and 4 were C4d+AAR and C4d-AAR, respectively. The development of DSA and late AMR were correlated. Median time to development of AMR was 1 year [16]. In the present time, the association between HLA-DQ an- tibodies and AMR was investigated by many study groups and numerous articles have been reported on this issue [17-19]. Willicombo et al. [19] have detected de novo DSA in 92 (18.2\%) of 505 cases in their study and $50(54.3 \%)$ of those cases were found to have HLA-DQ DSA. That study has identified a significant association between the presence of HLA-DQ DSA andAMR [19]. DeVos et al [20] have obtained similar outcomes and detected rejection in 21 of the cases with HLA-DQ antibody. The rate of 3-year graft survival was found statistically significantly lower in those patients [20]. HLA-DQ antibody was identified in 13 cases in our study. Only one patient was diagnosed with biopsy-proven AMR. However, diagnosis of subclinical AMR could not be established in the cases with HLA-DQ positivity since we did not perform routine renal biopsy. In our study, we determined the MFI cut-off values as 1000, 2000 and 4000. In our study, the relationship between these MFI values and AMR was not significant. There is no consensus on the MFI cut-off values. This value varies from clinic to clinic. Morris et al. [21]. They stated in their study that cases with MFI $<2000$ may not be an obstacle for transplantation. Also, in another study, a significant difference was observed between cases with MFI < 1500 and cases with $>1500$ in terms of graft survive [22].

Postoperative routine DSA monitoring in the patients with low immunological risk is not performed because of high-cost. However, it is a recommended procedure in the patients with high immunological risk. We have aimed to present a contribution to the studies that evaluated the association between routine DSA monitoring performed in our country and AMR.

\section{CONCLUSION}

Although, retrospective design of our study is a limitation, nevertheless, the fact that routine DSA monitoring is a high-cost procedure and that routine DSA monitoring in all the cases in our study provided no contribution to the outcomes of our study may contribute to the debate on necessity of routine DSA monitoring in the patients with low immunological risk.

\section{Authors' Contribution}

Study Conception: NA; Study Design: NA, VA; 
Supervision: NA, VA; Funding: NA, VA; Materials: NA, VA; Data Collection and/or Processing: NA, VA, ŞK; Statistical Analysis and/or Data Interpretation: NA, ŞK; Literature Review: NA; Manuscript Preparation: NA and Critical Review: NA, VA, ŞK.

\section{Conflict of interest}

The authors disclosed no conflict of interest during the preparation or publication of this manuscript.

\section{Financing}

The authors disclosed that they did not receive any grant during conduction or writing of this study.

\section{Acknowledgment}

This study was presented as an oral presentation at the 7th Current Kidney Diseases, Hypertension and Transplantation Meeting. 18-22 April, 2018, Bursa, Turkey

\section{REFERENCES}

1. Meier-Kriesche HU, Schold JD, Kaplan B. Long-term renal allograft survival: have we made significant progress or is it time to rethink our analytic and therapeutic strategies? Am J Transplant 2004;4:1289-95.

2. Morath C, Opelz G, Zeier M, Süsal C. Clinical relevance of HLA antibody monitoring after kidney transplantation. J Immunol Res 2014;2014:845040.

3. Loupy A, Hill GS, Nochy D, Legendre C. Antibody-mediated microcirculation injury is the major cause of late kidney transplant failure. Am J Transplant 2010;10:952.

4. Sellarés J, de Freitas DG, Mengel M, Reeve J, Einecke G, Sis $\mathrm{B}$, et al. Understanding the causes of kidney transplant failure: the dominant role of antibody-mediated rejection and nonadherence. Am J Transplant 2012;12:388-99.

5. Meier-Kriesche HU, Schold JD, Srinivas TR, Kaplan B. Lack of improvement in renal allograft survival despite a marked decrease in acute rejection rates over the most recent era. Am J Transplant 2004;4:378-83.

6. Terasaki PI. Humoral theory of transplantation. Am J Transplant 2003;3:665-73.

7. Lachmann N, Terasaki PI, Budde K, Liefeldt L, Kahl A, Reinke P, et al. Anti-human leukocyte antigen and donor-specific antibodies detected by luminex posttransplant serve as biomarkers for chronic rejection of renal allografts. Transplantation 2009;27;87:1505-13.

8. Einecke G, Sis B, Reeve J, Mengel M, Campbell PM, Hidalgo LG, et al. Antibody-mediated microcirculation injury is the major cause of late kidney transplant failure. Am J Transplant
2009;9:2520-31.

9. Sellarés J, de Freitas DG, Mengel M, Reeve J, Einecke G, Sis $\mathrm{B}$, et al. Understanding the causes of kidney transplant failure: the dominant role of antibody-mediated rejection and nonadherence. Am J Transplant 2012;12:388-99.

10. Li X, Ishida H, Yamaguchi Y, Tanabe K. Poor graft outcome in recipients with de novo donor-specific anti-HLA antibodies after living related kidney transplantation. Transpl Int 2008;21:1145-52.

11. McKenna RM, Takemoto SK, Terasaki PI. Anti-HLA antibodies after solid organ transplantation. Transplantation 2000;69:319-26.

12. Terasaki PI, Ozawa M. Predicting kidney graft failure by HLA antibodies: a prospective trial. Am J Transplant 2004;4:43843.

13. Süsal C, Aykut G, Morath C, Fichtner A, Unterrainer C, Scherer $\mathrm{S}$, et al. Relevance of donor-specific antibody monitoring after kidney transplantation: findings from the Collaborative Transplant Study and the Heidelberg Transplant Center. HLA 2019;94 Suppl 2:11-5.

14. Parajuli S, Joachim E, Alagusundaramoorthy S, Aziz F, Blazel J, Garg N, et al. Donor-specific antibodies in the absence of rejection are not a risk factor for allograft failure. Kidney Int Rep 2019;4:1057-65.

15. Cioni M, Nocera A, Innocente A, Tagliamacco A, Trivelli A, Basso S, et al. De novo donor-specific HLA antibodies developing early or late after transplant are associated with the same risk of graft damage and loss in nonsensitized kidney recipients. J Immunol Res 2017;2017:1747030.

16. Ginevri F, Nocera A, Comoli P, Innocente A, Cioni M, Parodi A, et al. Posttransplant de novo donor-specific HLA antibodies identify pediatric kidney recipients at risk for late antibody-mediated rejection. Am J Transplant 2012;12:3355-62.

17. Wiebe C, Gibson IW, Blydt-Hansen TD, Karpinski M, Ho J, Storsley LJ, et al. Evolution and clinical pathologic correlations of de novo donor-specific HLA antibody post kidney transplant. Am J Transplant 2012;12:1157-67.

18. Tagliamacco A, Cioni M, Comoli P, Ramondetta M, Brambilla C, Trivelli A, et al. DQ molecules are the principal stimulators of de novo donor-specific antibodies in nonsensitized pediatric recipients receiving a first kidney transplant. Transpl Int 2014;27:667-73

19. Willicombe M, Brookes P, Sergeant R, Santos-Nunez E, Steggar C, Galliford J, et al. De novo DQ donor-specific antibodies are associated with a significant risk of antibody-mediated rejection and transplant glomerulopathy. Transplantation 2012;94:172-7.

20. DeVos JM, Gaber AO, Knight RJ, Land GA, Suki WN, Gaber LW, et al. Donor-specific HLA-DQ antibodies may contribute to poor graft outcome after renal transplantation. Kidney Int 2012;82:598-604.

21. Morris GP, Phelan DL, Jendrisak MD, Mohanakumar T. Virtual cross match by identification of donor-specific anti-human leukocyte antigen antibodies by solid-phase immunoassay: a 30month analysis in living donor kidney transplantation. Hum Immunol 2010;71:268-73. 
22. Caro-Oleas JL, Gonza'lez-Escribano MF, Gonza'lez-Roncero FM, Acevedo-Calado MJ, Cabello-Chaves V, Gentil-Govantes MA, et al. Clinical relevance of HLA donor-specific antibodies detected by single antigen assay in kidney transplantation. Nephrol Dial Transplant 2012;27:1231-8. 